Check for updates

Cite this: J. Mater. Chem. C, 2020 8, 9688

Received 12th May 2020, Accepted 15th June 2020

DOI: $10.1039 / \mathrm{dOtc02295k}$

rsc.li/materials-c

\section{Near infrared organic photodetectors based on enhanced charge transfer state absorption by photonic architectures $\dagger$}

\author{
Martí Gibert-Roca, (D) Pau Molet, (D) Agustín Mihi (D) * and Mariano Campoy-Quiles (DD *
}

\section{Introduction}

Nowadays, near infrared (NIR) photodetectors are a ubiquitous technology, used on a daily basis in applications such as automatic sliding doors, NIR cameras, remote controls, telecommunications, spectrophotometers, and medical diagnosis. ${ }^{1,2}$ Current NIR photodetector technologies rely on combining silicon or, to a lesser extent, III-V (InGaAs) with color filters or gratings. ${ }^{3,4}$ This approach has proven to be functional and reliable, however, such wafer based photodetectors with extra filtering components fail to satisfy the new demands for wearable, cheap and flexible devices. In this direction, organic photodetectors (OPDs) are emerging as an appealing alternative, thanks to their flexibility, low embedded energy processing and high compatibility with available and easily scalable techniques such as roll to roll processing. ${ }^{5-8}$ Ease and cost of manufacture is not the sole advantage, since the composing organic polymers and/or small organic molecules, can be chemically tuned to provide a variety of absorption ranges. ${ }^{9-11}$ While this approach has worked well in the visible range, extending the photoresponse towards the NIR range has been challenging. Lower bandgap polymers and

Institut de Ciència de Materials de Barcelona (ICMAB-CSIC), E-08193 Cerdanyola del Vallès, Spain. E-mail: amihi@icmab.es, mcampoy@icmab.es, m.campoy@csic.es $\dagger$ Electronic supplementary information (ESI) available: Theoretical photonic analysis, and supplementary figures. See DOI: 10.1039/d0tc02295k small molecules with responses that go deeper into the infrared are being synthesized, ${ }^{12,13}$ however, these new materials are complex and expensive, both in time and energy, reducing their potential to be used in real devices. ${ }^{12}$ Moreover, new molecules have to be developed for each targeted wavelength range, strongly increasing the required synthetic efforts.

Alternatively, some effort has been devoted to extend and increase the absorption in the NIR region with inexpensive and readily available materials using new inventive designs. ${ }^{14,15}$ One approach that rendered promising results is the filtering effect in thick polymer layers, also known as charge collection narrowing, ${ }^{16}$ to obtain narrow and high external quantum efficiencies (EQE) in the NIR. However, since this approach relies on the intrinsic absorption of the materials, it still requires relatively low bandgap materials to reach into the NIR region.

A recently proposed elegant alternative is to take advantage of the direct intermolecular charge transfer state (CTS) absorption, extending the photoresponse into the NIR region without the need of low bandgap materials. ${ }^{17-20}$ The CTS is the result of the interaction between the highest occupied molecular orbital (HOMO) of the donor and the lowest unoccupied molecular orbital (LUMO) of the acceptor. This state appears only at the donor-acceptor interface, where the different electronic orbitals come together forming a new, intermediate state. The latter usually has a significantly lower transition energy than the corresponding constituents in a type II heterojunction, such as typical 
donor/acceptor blends. In other words, the HOMO energy level of the donor is higher than that of the acceptor and the LUMO energy level of the acceptor is lower than that of the donor (staggered gap). Since the CTS has a lower transition energy, the blend can potentially absorb light with photon energies below the bandgaps of each of its separate components. The CTS absorption strength depends on the intermixing of the molecules and, unfortunately, is around two orders of magnitude lower than singlet absorption due to the fact that it is an intermolecular state. ${ }^{13}$ The intrinsic low oscillator strength of the CTS often requires the use of very thick active layers at high reverse voltages in order to obtain a significant photoresponse. ${ }^{21}$ In a traditional device configuration, in order to enhance the absorption of the CTS, the thickness of the active layer must be increased enormously (tenths of microns); this implies the usage of hundreds of Volts applied bias to extract charges efficiently. ${ }^{21}$ In order to avoid such high voltages and thick layers, light trapping schemes offer an interesting alternative to greatly enhance the CTS absorption. Recently, the use of metal cavities has led to an impressive photoresponse in OPDs beyond the bandgaps of the composing materials. ${ }^{2-25}$ When using optical micro-cavities the detection wavelength can be tuned by accurately controlling the cavity dimensions, i.e., the active layer thickness. While these devices exhibit a very good performance, the resonant frequency critically depends on film thickness, thus they may not be compatible with techniques such as roll to roll, in which thickness usually fluctuates within a certain tolerance.

Inspired by the micro-cavity devices, in this work we propose a device configuration that will enhance the CTS absorption effect via a nanostructured active layer in the shape of a photonic crystal. ${ }^{26}$ Major accomplishments have been achieved already in photovoltaics by structuring the device with photonic crystals. $^{27-30}$ In the case of $2 \mathrm{D}$ photonic crystals, its periodic structure diffracts incident light and enhances the optical path within the active layer, thus rendering higher efficiencies. In this work we will provide our photodetector with a back-electrode structured as a 2D photonic crystal engineered to couple incident light to photonic modes with enhanced electric field concentration in the active medium at those wavelengths of interest for the CTS absorption effect. To fulfill real application demands, the designed device has been fabricated with low cost blend materials using inexpensive and scalable techniques such as blade coating for the photodetector manufacturing and nanoimprinting softlithography for the active layer nanostructuration. The active layer blends are composed of a bulk heterojunction (BHJ) of an electron donor PBTTT or P3HT and an electron acceptor $\mathrm{PC}_{71} \mathrm{BM}$ or $\mathrm{PC}_{61} \mathrm{BM}$. We show that these active layers can be easily and seamlessly nanostructured via soft nanoimprinting lithography, enhancing the EQE response from 750 to $1000 \mathrm{~nm}$ and from 775 to $1075 \mathrm{~nm}$, for P3HT:PC ${ }_{61} \mathrm{BM}$ and PBTTT: $\mathrm{PC}_{71} \mathrm{BM}$, respectively. We attribute this photoresponse, significantly beyond the band gap of the active layer, to the absorption of the CTS. Different photonic lattice parameters have been used to enhance the absorption at specific wavelengths, and thus to provide wavelength tuneability on the NIR region. Furthermore, since the enhanced photodetection wavelength is thickness independent, the processing tolerances can be higher, making this device attractive for further development and commercialization. In summary, the designed light trapping configuration implemented enables us to use inexpensive organic materials for photodetection into the NIR with minimal disruption of the fabrication process and where the nanostructuring step does not negatively affect the electrical performance of the photodetector.

\section{Results and discussion}

\subsection{Device fabrication}

The organic NIR photodetectors were fabricated from P3HT: $\mathrm{PC}_{61} \mathrm{BM}$ and PBTTT:PC ${ }_{71} \mathrm{BM} \mathrm{BHJ}$ blends (chemical structure in Fig. 1a) for three main reasons: first, they are inexpensive materials, which makes them appealing for large scale applications; second, they exhibit a significant CTS absorption that extends into the NIR region, so we can extend the response of the devices beyond their band gaps; and third, they have sufficiently high carrier mobilities that allow us to deposit thick active layers, which is essential to take maximum profit of the CTS low absorption coefficient. $^{13}$

First, we deposited a ZnO electron transport layer (ETL) over the ITO covered glass substrates by blade coating a technique that is often used as a pre-upscaling method.

After annealing the film, we deposited a thick layer of active material also by blade coating. Then, we nanostructured the dry active layer via soft nanoimprinting lithography (Fig. 2a). This technique is reliable, cleanroom free, inexpensive and can produce extended nanostructured areas with high fidelity, plus it is highly scalable and compatible with roll to roll processes. ${ }^{31,32}$

Moreover, this non-disruptive technique avoids using aggressive nanostructuring processes, such as reactive ion etching, that could deteriorate the electrical properties of the device. Pressing a pre-patterned PDMS (polydimethyl siloxane) stamp mold onto the active layer at a temperature over the glass transition of the polymer blend is enough to produce the negative photonic crystal imprint on the device (see Experimental section). Our pattern consists of a square array of cylindrical pillars that acts as a diffraction grating, coupling the incident light into propagating modes of the active layer. We used Finite-Differences Time-Domain method (FDTD) to engineer the optimal photonic structures for our active materials and provide insight on the light propagation within the device (Fig. S1, ESI $\dagger$ ). We concluded that, array lattice parameters $(L)$ of 400,500 and $600 \mathrm{~nm}$ (from now on also referred to as $L=400, L=500$ and $L=600$ ) place their first orders of diffraction inside the range of absorption of the CTS. This would then provide efficient light trapping at NIR frequencies below the band gap of the $\mathrm{BHJ}$ materials, thus amplifying the CTS absorption. The thermally evaporated $\mathrm{MoO}_{3}$ layer thickness was set to $10 \mathrm{~nm}$, enough to have a uniform film with good electrical performance while maintaining the optical properties of the device (Fig. S2, ESI $\dagger$ ). Finally, a thick $200 \mathrm{~nm}$ $\mathrm{Ag}$ back electrode contact was evaporated onto the samples followed by a thermal annealing to increase their crystallinity 
a)

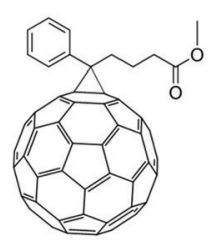

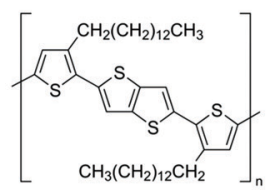
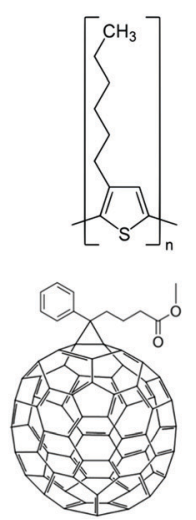

b)

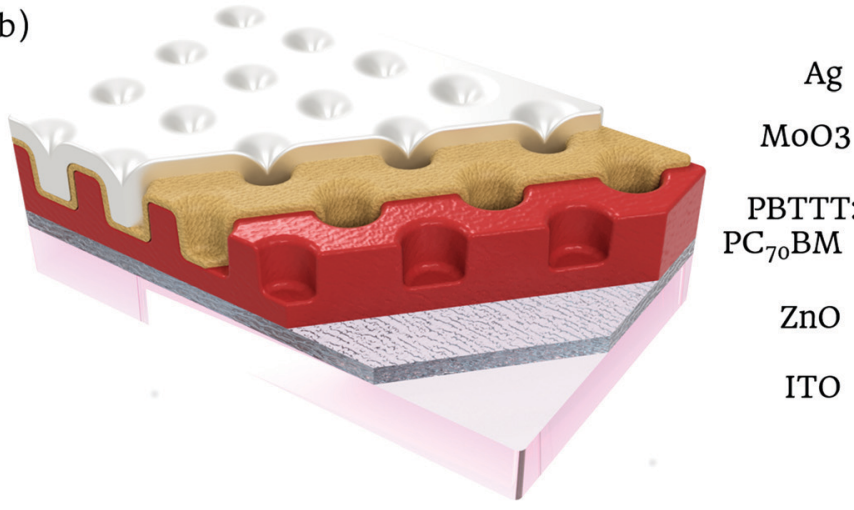

Fig. 1 Schematics of the nanostructured organic NIR photodetector. (a) Phenyl- $C_{61}$-butyric acid methyl ester $\left(\mathrm{PC}_{61} \mathrm{BM}\right)(>99.5 \%)$, poly $(3-$ hexylthiophene-2,5-diyl) (P3HT), [6,6]-phenyl $C_{71}$ butyric acid methyl ester ( $\left.\mathrm{PC}_{71} \mathrm{BM}\right)$ and poly[2,5-bis(3-tetradecylthiophen-2-yl)thieno[3,2-b]thiophene] (PBTTT-C14). (b) Diagram the organic photodetector structure and each layer's material.

a)
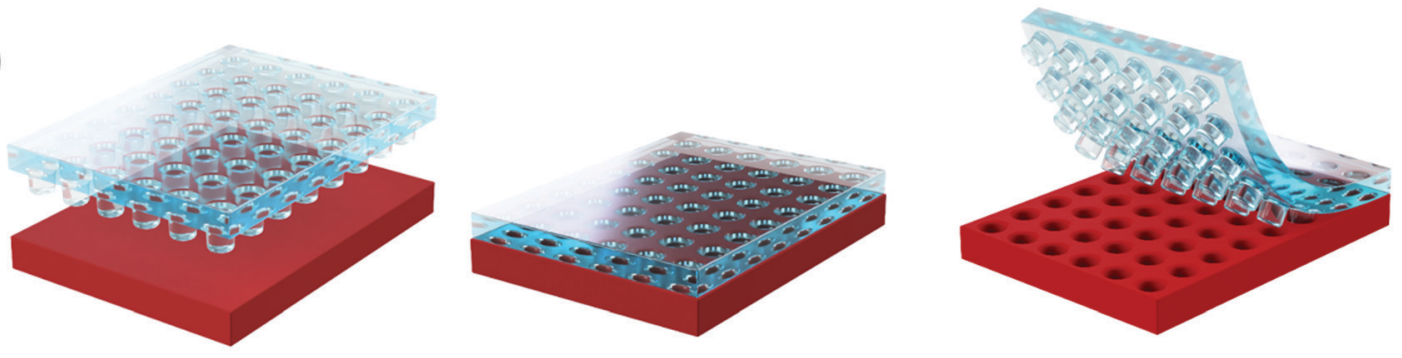

b)

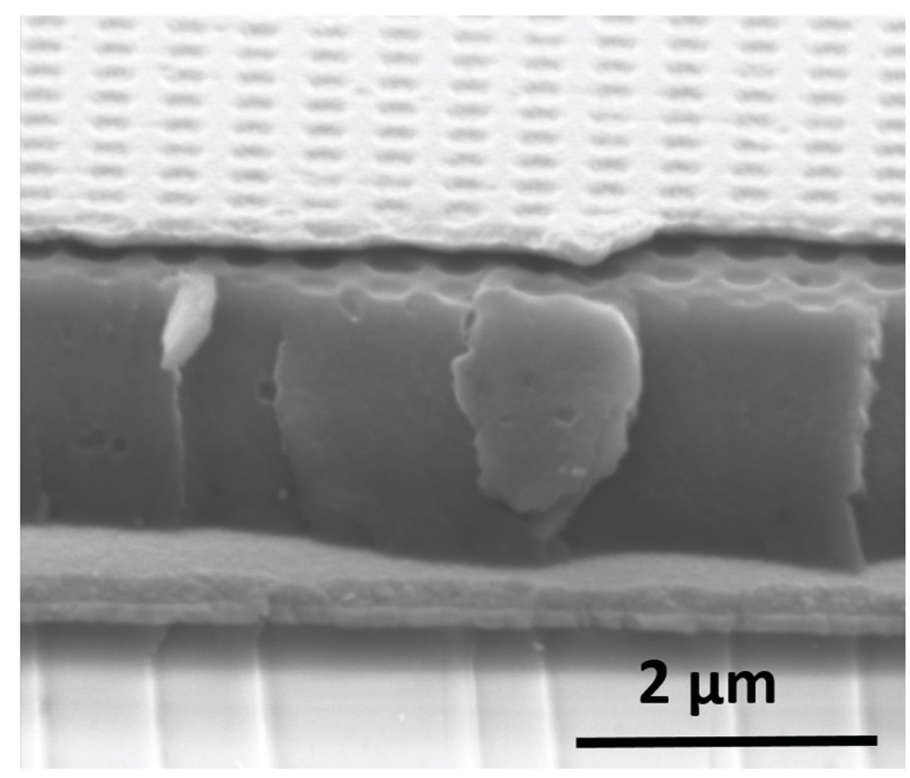

c)

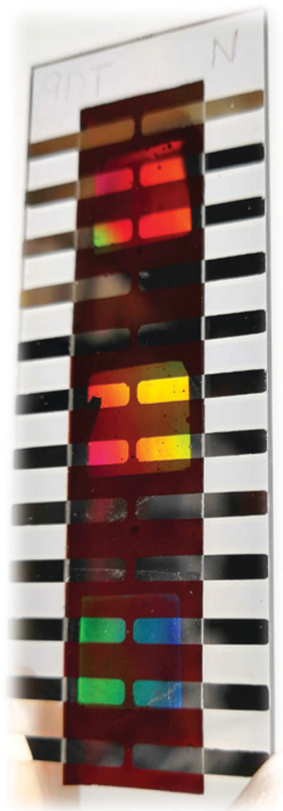

Fig. 2 (a) Soft nanoimprinting lithography process steps. (b) SEM image of one of the finished nanostructured photodetectors. (c) Picture of the finished device showing different iridescence for each nanostructuration; $L=600, L=500, L=400$ from top to bottom.

(Fig. $2 \mathrm{~b}$ and c). Since the back electrode was deposited onto the nanostructured active layer, it followed the corrugation resulting in a nanostructured back electrode (Fig. 2b) with a strong optical response (Fig. S3, ESI†).

\subsection{Proof of concept photonic NIR device}

The electrical characterization of our devices starts with EQE measurements. The EQE measurements of the devices with the photonic back electrode revealed several peaks in the NIR 


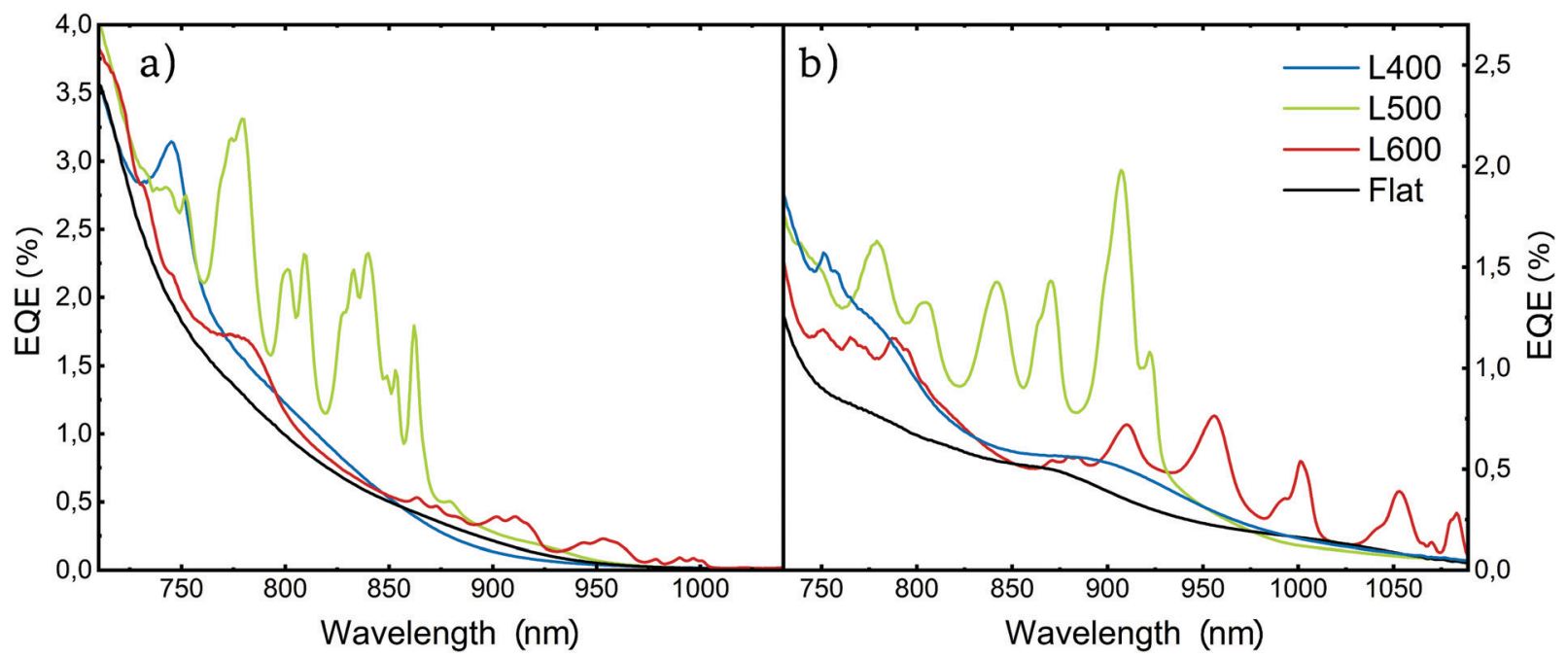

Fig. 3 External quantum efficiency measurements for non-structured (flat) and differently nanostructured photodetectors $(L=400, L=500$ and $L=$ 600 ) with an active layer of (a) P3HT:PC ${ }_{61} B M$, (b) PBTTT:PC ${ }_{71} B M$

region that were absent in the reference flat samples (Fig. 3), for both active layer compositions.

As predicted by our simulations, the spectral range at which this enhanced EQE appears depends on the lattice parameter of the $2 \mathrm{D}$ array and the refractive index of the active layer. Both larger lattice parameters and higher active layer refractive indexes induce a redshift in the position of the peaks (Fig. 3b and a respectively).

For both active layer compositions, the devices with the smallest lattice parameter of $400 \mathrm{~nm}$ present a narrow peak at the tail of the singlet absorption (Fig. $3 \mathrm{a}$ and $\mathrm{b}$ blue lines) located at $750 \mathrm{~nm}$ for $\mathrm{P} 3 \mathrm{HT}: \mathrm{PC}_{61} \mathrm{BM}$ and two small peaks between $750 \mathrm{~nm}$ and $775 \mathrm{~nm}$ for PBTTT:PC ${ }_{71} \mathrm{BM}$. This represents an enhancement in $\mathrm{EQE}$ of $60 \%$ and $70 \%$ for each blend respectively (Fig. S4 and S5, ESI $\dagger$ ).

The devices imprinted with nanostructures with $L=500 \mathrm{~nm}$ exhibit several peaks in the EQE into the NIR range below the bandgap of both blends. The EQE reaches over $2 \%$ for both PBTTT:PC ${ }_{71} \mathrm{BM}$ and P3HT:PC ${ }_{61} \mathrm{BM}$ (Fig. $3 \mathrm{a}$ and $\mathrm{b}$ green lines). The P3HT: $\mathrm{PC}_{61} \mathrm{BM}$ devices exhibit photoresponse in the $800-$ $850 \mathrm{~nm}$ region (Fig. 3a), with a 4-fold enhancement factor at its maximum at $837 \mathrm{~nm}$ (Fig. S4, ESI $\dagger$ ) with respect to the planar reference, achieving a maximum EQE value of $2.4 \%$. In the case of PBTTT:PC ${ }_{71} \mathrm{BM}$, the EQE shows peaks deeper into the NIR $(850-920 \mathrm{~nm})$, where a 5 -fold increase on conversion efficiency can be seen at $907 \mathrm{~nm}$, with EQE maximum values of $2 \%$ (Fig. 3b and Fig. S5, ESI $\dagger$ ).

Finally, those devices incorporating the $L=600 \mathrm{~nm}$ array show EQE enhancement peaks with maximum values around $0.5 \%$, deep into the NIR reaching wavelengths of $1000 \mathrm{~nm}$ and almost $1100 \mathrm{~nm}$ for P3HT:PC ${ }_{61} \mathrm{BM}$ and PBTTT:PC ${ }_{71} \mathrm{BM}$, respectively (Fig. $3 \mathrm{a}$ and $\mathrm{b}$ red lines). It is worth noting that the EQE performance is lower for $L=600 \mathrm{~nm}$ compared to other lattice configurations.

This is mainly due to the lower values of the CTS absorption coefficient at higher wavelengths, being more pronounced in P3HT:PC ${ }_{61} \mathrm{BM}$ than in PBTTT:PC ${ }_{71} \mathrm{BM}^{25}$ In P3HT:PC ${ }_{61} \mathrm{BM}$ based devices, the EQE peaks, for $L=600 \mathrm{~nm}$, are located around $900-1050 \mathrm{~nm}$, with a local maximum at $1000 \mathrm{~nm}$ where a 5.8-fold enhancement is reached (Fig. S4, ESI $\dagger$ ). In the case of PBTTT:PC ${ }_{71} \mathrm{BM}$, the devices show photoresponse at the 950$1100 \mathrm{~nm}$ region, for the same lattice parameter, where the local maximum enhancement, located at $1083 \mathrm{~nm}$, is 6 times better than its flat counterpart (Fig. S5, ESI $\uparrow$ ).

We illustrate the optical light trapping scheme responsible for the enhanced response in the devices for the case of a $500 \mathrm{~nm}$ lattice parameter nanostructure (Fig. 4). The calculated absorption spectrum (Fig. 4a) of the $L=500 \mathrm{~nm}$ PBTTT:PC ${ }_{71} \mathrm{BM}$ device is in good agreement with the measured external quantum efficiency spectrum (Fig. 4b) allowing us to validate the effect of the photonic architecture through the examination of the spatial distribution of the electric fields at the wavelengths of maximum response. The electric field profile at $862 \mathrm{~nm}$ and $907 \mathrm{~nm}$ is presented for both the nanostructured (Fig. 4c) and the flat (Fig. 4d) devices. The fields in the planar device present a standard plane wave distribution (Fig. $4 \mathrm{f}$ and h), while the patterned devices show a diffraction pattern inside the active layer with field enhancements over 6 times the incident power (Fig. 4e and g). In sum, the enhanced optical path in the active layer, generated by the diffraction modes of the array, provides a higher absorption of the CTS at the selected wavelengths.

\subsection{Additional design rules}

In this section, we explore the influence of additional geometrical parameters on the performance of the devices. We first start by looking at the thickness of the active layer (Fig. S6 and S7, ESI $†$ ). Two main observations can be made. First, the enhanced frequencies are mainly given by the lattice parameter, almost independently of the active layer thickness (in the range considered here). This is a remarkable difference with respect to micro-cavitybased photodetectors. ${ }^{25}$

Second, the optimum active layer thickness in terms of the enhancement factor, arising from the compromise between the 
a)

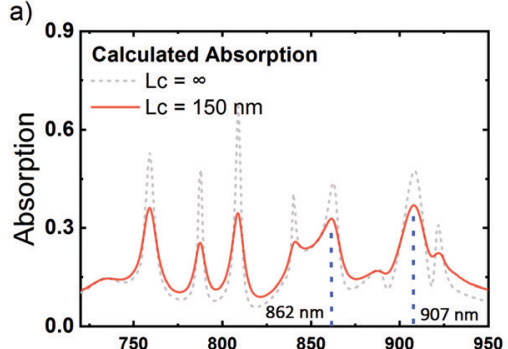

b)

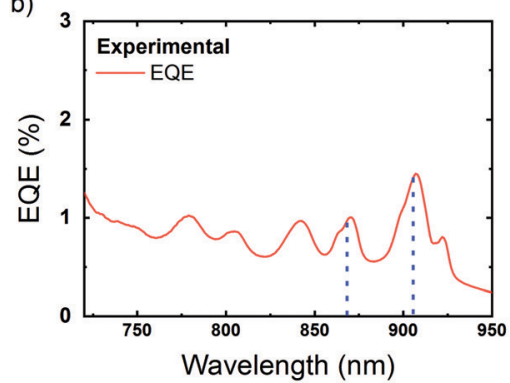

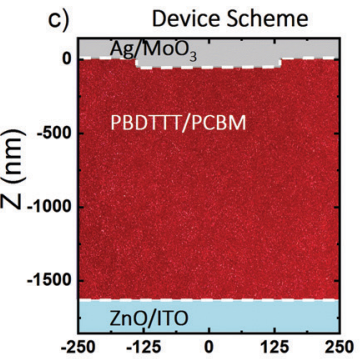

d)

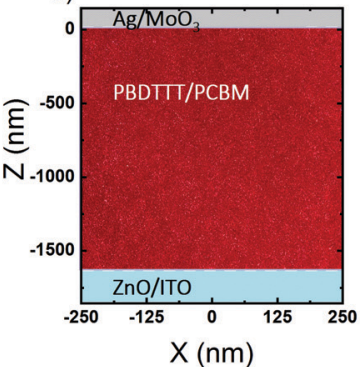

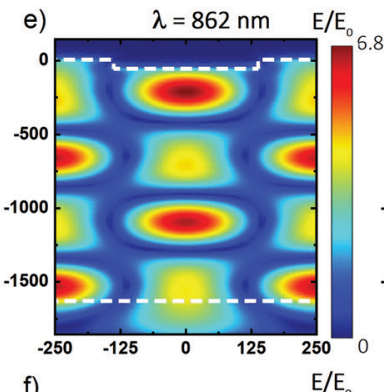

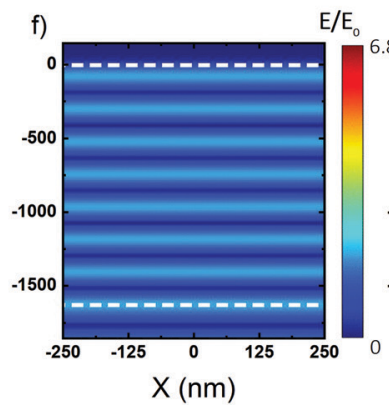

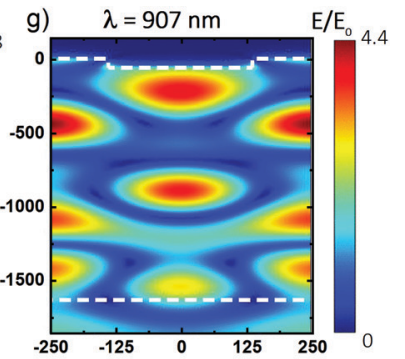

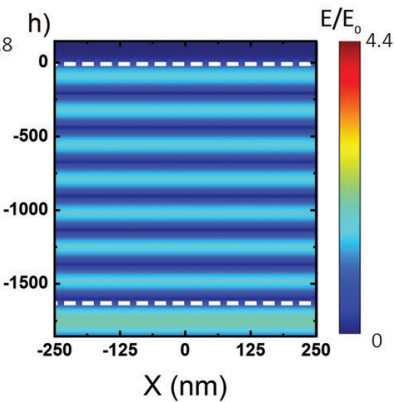

Fig. 4 (a) Calculated absorption of the fabricated devices with fully coherent light (grey dashed) and 150 micron coherent light (red). (b) External quantum efficiency measurements for a PBTTT:PC ${ }_{71} B M$ nanostructured photodetector with a lattice parameter of $L=500 \mathrm{~nm}$. Sample scheme for nanostructured and flat devices (c), (d) and calculated electric fields at wavelengths 862 and $907 \mathrm{~nm}(\mathrm{e})$, (f) and (g), (h).

optical and electrical properties of the device. Thin active layers (on the order of $100 \mathrm{~nm}$ ) exhibit high EQEs in the visible but negligible CTS absorption in the NIR. Thicker active layers exhibit enhanced CTS absorption, but often deteriorated electrical response. This is due to the non-uniform charge generation distribution across the film thickness, which implies that the travel path for half of the charges is significantly longer, leading to high recombination probabilities, and finally decreasing the overall electrical response. ${ }^{16}$ We have found optimum active layer thicknesses of $700 \mathrm{~nm}$ for P3HT: $\mathrm{PC}_{61} \mathrm{BM}$ and of $1600 \mathrm{~nm}$ for PBTTT:PC ${ }_{71} \mathrm{BM}$. The optimum thickness is higher for PBTTT because of its higher mobility with respect to P3HT. ${ }^{33}$

Another important parameter to take into account when designing the nanostructure is the depth of the array of holes imprinted on the active layer. Deeper holes lead to features exhibiting stronger diffraction and thus provide more efficient light trapping. However, if the imprinted holes are too deep, the $\mathrm{MoO}_{3}$ electron blocking layer that is evaporated on top may not form a continuous film, leading to pinholes that disrupt the electrical properties of the device (e.g. unacceptably high dark currents). For this reason, we limited the height of the features in our architecture to $60 \mathrm{~nm}$, avoiding pinhole generation with a complete coverage of the active layer by each of the subsequent layers. This effect is illustrated by comparing devices with different $\mathrm{MoO}_{3}$ layer thicknesses in Fig. S2 (ESI $\dagger$ ).

The active layer morphology is often a very important parameter for the performance of organic based diodes. ${ }^{33-36}$ While a full study of morphological effects on the response of NIR photodiodes goes beyond the scope of this manuscript, we have evaluated the role of thermal annealing on the EQE of the devices. Indeed, we have found that annealing the samples has a significant effect on its performance, increasing its response by 10-20 times (Fig. S8, ESI $\dagger$ ).

\subsection{Advanced photodiode characterization}

In this section, we show the performance of the photonic NIR photodetectors as a function of bias, illumination, time response as well as illustrating the spatial homogeneity of the performance.

First, we start by evaluating the photodetectors under reverse $\mathrm{V}$ bias to evaluate the charge generation and collection capacity of our nanostructured devices compared to the flat reference samples. The photodetector with the imprinted $L=$ $500 \mathrm{~nm}$ structure on the PBTTT:PC ${ }_{71} \mathrm{BM}$ active layer presented an increase in EQE from $2 \%$ to $7.5 \%$ at $907 \mathrm{~nm}$ while the reference device still lays below $2 \%$ under a $23 \mathrm{~V}$ reverse bias (Fig. 5a and b). At this high reverse bias, practically all charges generated are extracted, further improving the charge extraction from CTS. This is translated into a 4 times efficiency increase in our photonically enhanced devices under reverse bias, compared to the

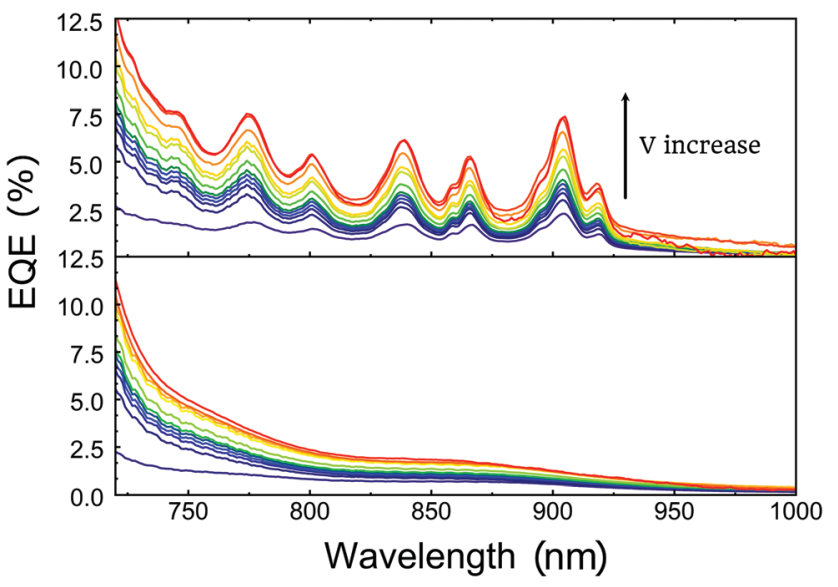

Fig. 5 EQE measurements with various reverse $\mathrm{V}$ bias from $0 \mathrm{~V}$ to $23 \mathrm{~V}$ for (a) nanostructured and (b) non-structured (flat) PBTT: $\mathrm{PC}_{71} \mathrm{BM}$ photodetectors. 
unbiased case. The saturation of the charge extraction, upon applying a reverse $\mathrm{V}$ bias, occurs much earlier in planar devices $(12 \mathrm{~V})$ than in our imprinted devices $(28 \mathrm{~V})$ confirming the important difference in charge generation between our nanostructured photodetector and the reference one (Fig. 6a). At high applied bias, the photoresponse of the device is only limited by the absorption of the material, since the bias helps to dissociate charges. It is here where the effect of the improved absorption in the photonic case is most evident.

This enhancement can also be observed on the responsivity of the photodetectors, where the $L=500 \mathrm{~nm}$ PBTTT:PC ${ }_{71} \mathrm{BM}$ device shows a responsivity of $15 \mathrm{~mA} \mathrm{~W}^{-1}$ in contrast with the $3 \mathrm{~mA} \mathrm{~W}^{-1}$ for their flat counterpart at $907 \mathrm{~nm}$. (Fig. S9, ESI $\dagger$ ). When a reverse $\mathrm{V}$ bias is applied the responsivity also increases significantly reaching $54 \mathrm{~mA} \mathrm{~W}^{-1}$ for the nanostructured devices at $23 \mathrm{~V}$ versus the response of $9 \mathrm{~mA} \mathrm{~W}^{-1}$ of their flat counterparts at the same voltage (Fig. 6a).

While the performance of our OPDs is one order of magnitude lower than that of singlet absorption based OPDs, the values obtained are on the same order of magnitude as other OPDs based on similar strategies, such as the ones reported by Meredith et $a{ }^{16}{ }^{16}$ Also they exhibit a similar performance on the NIR to previously reported CTS based photodetectors that worked under high reverse $\mathrm{V}$ biases of $200 \mathrm{~V} .^{21,37}$

Nanoimprinted and flat devices exhibit similar dark currents, indicating that we successfully avoided pinhole formation and have a good conformal layer deposition (Fig. 6b). Obtained values are around $10 \mathrm{nA} \mathrm{cm}{ }^{-2}$ at $1 \mathrm{~V}$ and $1 \mu \mathrm{Acm}^{-2}$ at $15 \mathrm{~V}$. We consider this dark current values low considering the large area of photodetection $\left(8 \mathrm{~mm}^{2}\right)$. Taking into account the fact that, especially with solution processing, dark current does not scale up on a linear fashion. That meaning that maintaining low current densities at high areas is much more difficult due to higher probability of having defects on the production of larger devices.

Thanks to these low dark currents, the on/off ratio of our nanostructured photodetectors at $3.5 \mathrm{~mW} \mathrm{~cm}^{2}$ is around 850 for $L=500 \mathrm{~nm}$ at $-1 \mathrm{~V}$ and $907 \mathrm{~nm}$, being 5.5 times higher than in flat photodetectors (150). These values are comparable to other organic photodiodes, such as those reported by Zhu et al. and Jahnel et $a l .{ }^{38,39}$ For $L=600 \mathrm{~nm}$ photodetectors, the on/off ratio decreases down to 15 at a wavelength of $1080 \mathrm{~nm}$ and under $1 \mathrm{~V}$ of reverse bias, but they still outperform their flat counterparts, with on/off ratios of 3 at the same conditions. Under higher reverse biases the on/off ratio is still consistently superior for the nanostructured devices when compared with their flat reference (Fig. S10, ESI $\dagger$ ). While a full frequency response study has not been performed, the transient photocurrent experiments revealed that these devices show rise times on the order of microseconds at $0 \mathrm{~V}$ bias, for both nanostructured and flat devices as shown in Fig. 6c. These results show that the nanostructure does not significantly affect the frequency response of the devices.

The response of the nanostructured photodetectors is highly linear as illustrated by the linear dynamic range measurement of the $L=500$ structure at $907 \mathrm{~nm}$, which exhibits $r^{2}$ values of
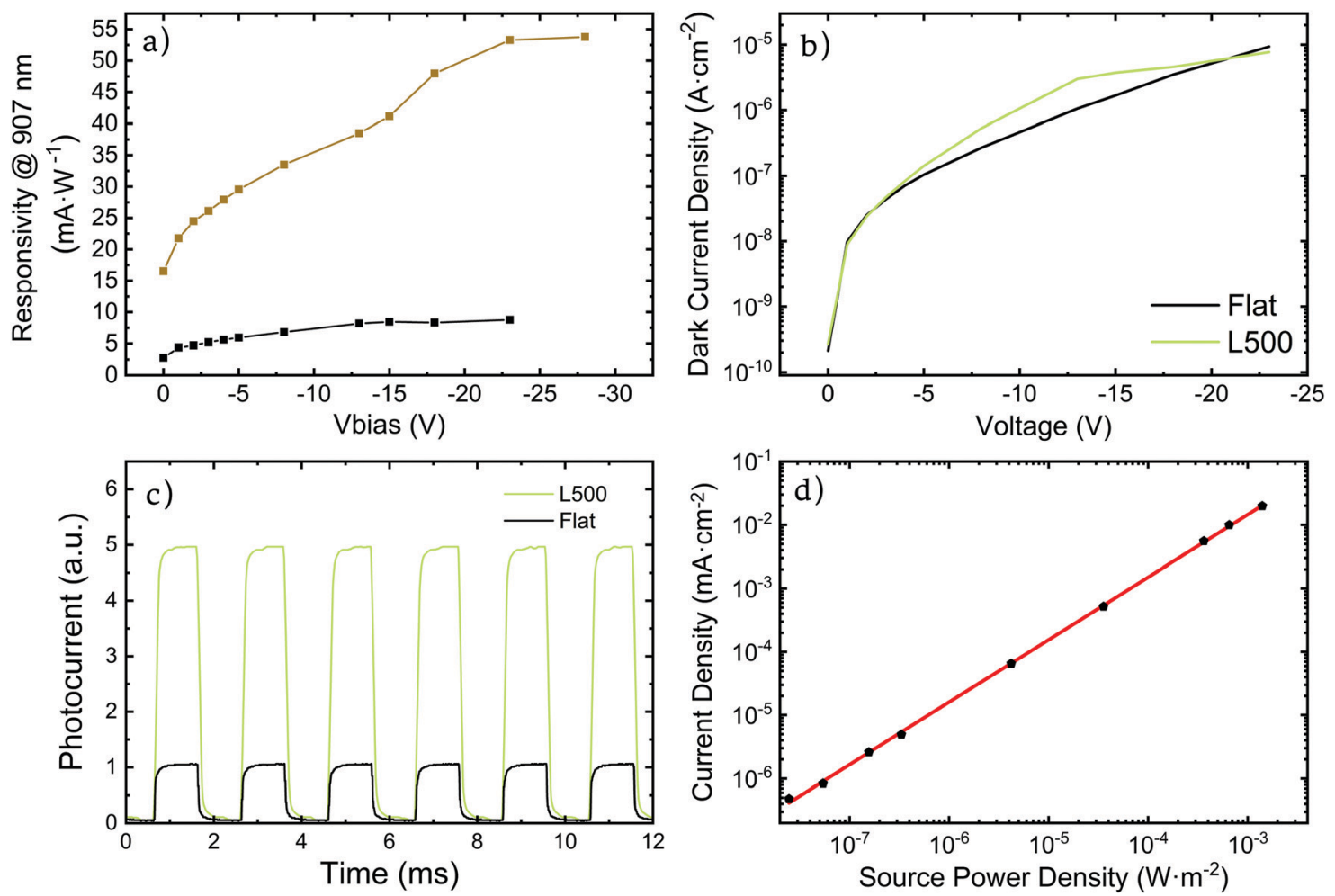

Fig. 6 (a) Responsivity at $907 \mathrm{~nm}$ for different $V$ bias of $L=500$ (nanostructured) and non-structured (flat) PBTTT:PC ${ }_{71} B M$ photodetector. (b) Dark current of both flat and $L=500$ nanostructured devices at various reverse $V$ bias. (c) Time dependent normalized photoresponse measurement for both flat and $L=500$ nanostructured devices at $0 \mathrm{~V}$ bias. (d) Linear dynamic range of PBTTT:PC ${ }_{71} B M$ nanostructured photodetector. 
a)

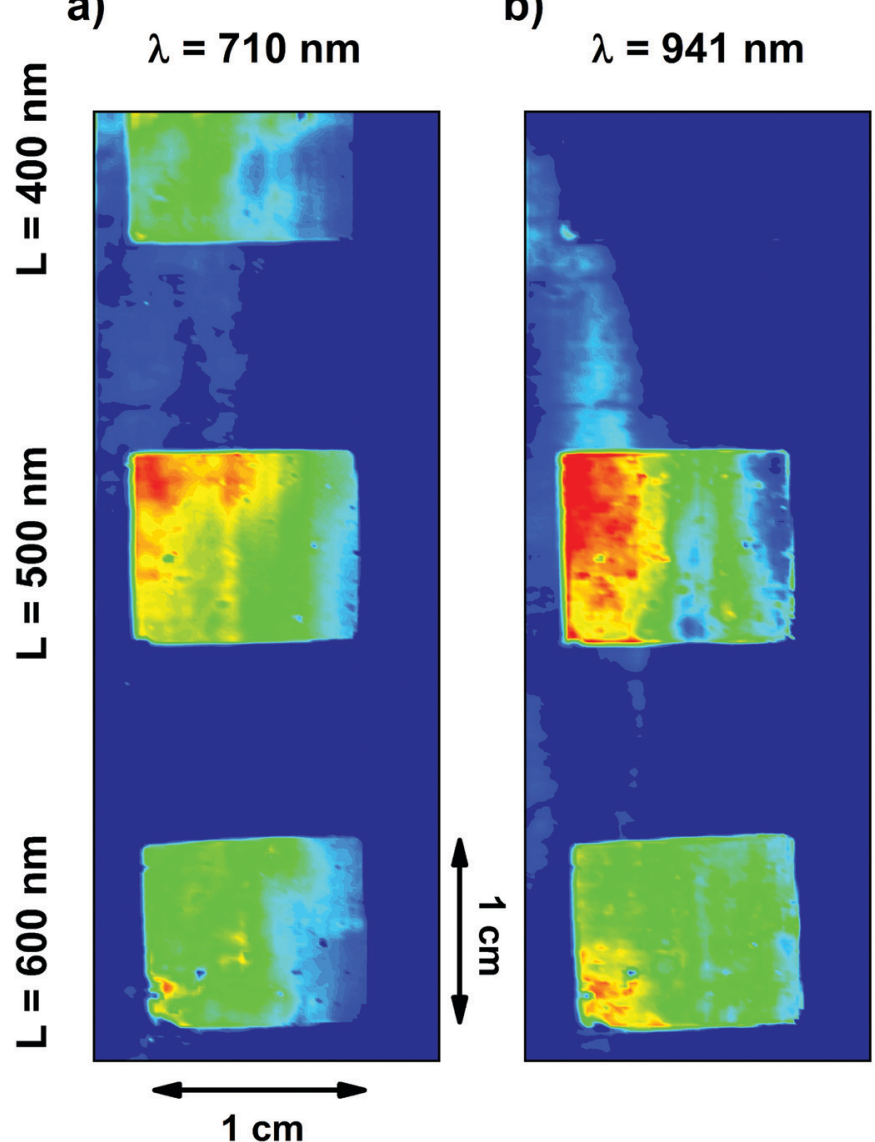

c) $\lambda=1032 \mathrm{~nm} \quad \mathrm{EQE}$ (norm.)

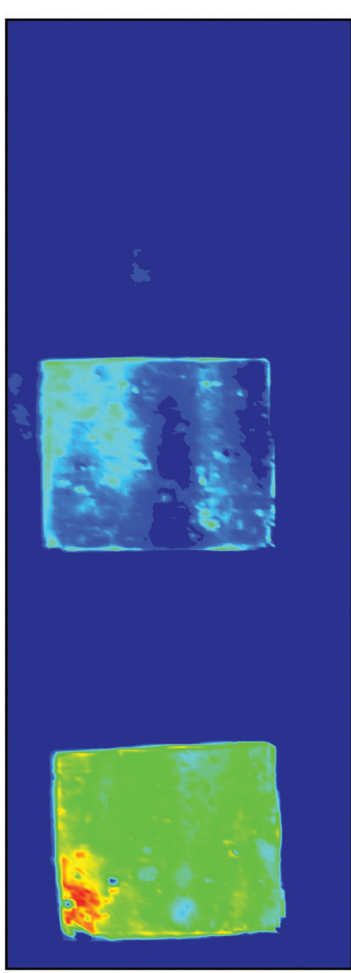

0.999 over more than $50 \mathrm{~dB}$ and a slope of 0.98565 , in the whole range available to our set up (Fig. 6d). The fact that the slope is so close to one guarantees a linear response of the photodetector on the given range.

Finally, in order to evaluate the spatial homogeneity of the devices, we fabricated large area devices with patterned areas of $1 \mathrm{~cm}^{2}$ surrounded by flat regions and we performed light beam induced photocurrent (LBIC) mapping. For this, we used a PBTTT:PC ${ }_{71}$ BM sample which included areas structured with three different lattice parameters. The photocurrent maps in Fig. 7 clearly demonstrate that the photocurrent is enhanced where the active layer has been structured. Moreover, the $\mathrm{cm}$ squared areas are relatively homogeneous, with the modulations in photocurrent intensity arising from small thickness variations produced during the blade coating of an entire microscope slide.

The inspection of the devices at three different wavelength excitations showed how the absorption of the CTS depended greatly on the lattice parameter, in agreement with the EQE data (Fig. 3). When excited with a wavelength of $710 \mathrm{~nm}$, all three patterns offer similar responses because they all provide diffraction at these wavelengths, where the singlet tail absorption is still present (Fig. 7a). Although no significant difference is observed between the three patterns, they all outperform the flat non-patterned regions that can be seen in between the patterns, revealing the importance of the nanostructuration. When the excitation wavelength is set to $941 \mathrm{~nm}$, where there is no contribution of the singlet tail absorption, only CTS absorption, the difference between the three fabricated structures becomes more relevant (Fig. 7b). At this wavelength, the pattern of $L=400 \mathrm{~nm}$ does not provide diffraction orders capable to enhance the absorption the CTS and produce a noticeable photocurrent, so its response is equal to the flat zones.

Meanwhile, regions with $L=600 \mathrm{~nm}$ and $500 \mathrm{~nm}$ still show good photocurrents, being the last one the optimum for this wavelength, as studied before with the spectral EQE. Finally, at $1031 \mathrm{~nm}$, only the region patterned with the $600 \mathrm{~nm}$ lattice parameter offers good enhancement with respect to the planar reference.

Interestingly, the response for each lattice parameter presents a series of peaks. For a given wavelength, each effective pixel has a different response. If each pixel is calibrated using the EQE data, one could think of a spectrometer based on three pixels, emulating the RGB strategy, but for the NIR. Fig. 7 can be considered, therefore, as an artificial NIR eye.

\section{Conclusions}

We report a new organic $\mathrm{BHJ}$ based photodetector architecture that is capable of detecting light at wavelengths significantly 
below the bandgap of its components. This was achieved by boosting the absorption of the long CTS absorption tail present in P3HT:PC ${ }_{61} \mathrm{BM}$ and PBTTT:PC ${ }_{71} \mathrm{BM}$ blends. Their CTS absorption was enhanced by incorporating a photonic architecture within the device via the nanostructuration of the active layer. Both the active layer and the patterning are carried out using roll to roll compatible and highly scalable techniques. The wavelength at which the photodetectors response is enhanced proved to be quite insensitive to thickness variations, offering appealing fabrication tolerances.

Furthermore, the used polymers and small molecules are inexpensive and easy to produce, making them ideal for large scale production. The performance of the photodetectors was tested through several techniques, showing that our nanostructured OPDs are significantly superior than flat OPDs on the NIR thanks to our strategy to enhance the CTS absorption. In addition, we demonstrated that different nanostructuration configurations enhance the response of the photodetectors at different wavelengths, rendering any of our devices wavelength tunable within a wide region on the NIR. In summary, we have developed wavelength tunable NIR photodetectors exploiting the CTS absorption, which opens new opportunities for the operation of organic based devices beyond their bandgaps.

\section{Experimental section}

\subsection{Materials and devices}

Poly(3-hexylthiophene-2,5-diyl) (P3HT) (99.995\%) (regioregular 95\%, average $\left.M_{\mathrm{n}} 54000-75000\right)$ was bought from Sigma Aldrich. Phenyl- $\mathrm{C}_{61}$-butyric acid methyl ester $\left(\mathrm{PC}_{61} \mathrm{BM}\right)(>99.5 \%)$ and $[6,6]$-phenyl $\mathrm{C}_{71}$ butyric acid methyl ester $\left(\mathrm{PC}_{71} \mathrm{BM}\right)(>99 \%)$, were purchased from OSSILA.

We have manufactured two main types of photodetectors; either with the pixelated substrates or with a continuous ITO back electrode, to be used in the EQE and LBIC measurements respectively.

On top of a pre-cleaned ITO covered glass substrate, we have deposited the $\mathrm{ZnO}$ electron transport layer from a nanoparticle solution (Avantama N-10) via Blade coating (Zehntner ZAA 2300). The parameters we have used are the following: droplet volume $50 \mu \mathrm{l}$, blade gap $50 \mu \mathrm{m}$, blade temperature $40{ }^{\circ} \mathrm{C}$ and speed $5 \mathrm{~mm} \mathrm{~s}^{-1}$. After the layer had been deposited we anneal it at $100{ }^{\circ} \mathrm{C}$ to fully dry and improve crystallinity. This procedure was repeated four times. Each time the deposition was performed on the opposite direction to the prior deposition. After this step, we proceeded to deposit the active layer, for the two different mixtures, the P3HT: $\mathrm{PC}_{61} \mathrm{BM}(1: 0.8)$ and the PBTTT: $\mathrm{PC}_{71} \mathrm{BM}(1: 4)$ (30 $\mathrm{mg} \mathrm{ml}^{-1}$ ) which had been dissolved in a mixture of chlorobenzene and dichlorobenzene $(1: 1)$. It was deposited via blade coating, with the following parameters: droplet volume $50 \mu \mathrm{l}$, blade gap $50 \mu \mathrm{m}$, blade temperature $90{ }^{\circ} \mathrm{C}$ and speed $84 \mathrm{~mm} \mathrm{~s}^{-1}$. The active layer was also deposited four times sequentially on opposite directions each time. Afterwards, the active layer was nanostructured via Nanoimprinting lithography, where we pressed with a prepatterned PDMS stamp at 4 bars while heating at $135{ }^{\circ} \mathrm{C}$ under vacuum conditions. This procedure was done with a Nanoimprinting Lithography setup (CNI v2.1 from NIL technologies). Afterwards we gently and slowly pulled the PDMS stamp away from the substrate, revealing the nanostructured surface that was visibly iridescent. On top of the active layer we deposited a layer of $10 \mathrm{~nm}$ of $\mathrm{MoO}_{3}$ by thermal evaporation at a rate of $0.1 \mathrm{~nm} \mathrm{~s}^{-1}$. And then we evaporated a back electrode of $200 \mathrm{~nm}$ of silver at an initial rate of $0.1 \mathrm{~nm} \mathrm{~s}^{-1}$ until $40 \mathrm{~nm}$ and then at a rate of $0.3 \mathrm{~nm} \mathrm{~s}^{-1}$. Later on we proceed to encapsulate the samples with a glass coverslip and epoxy resin. Finally, we annealed the samples at $135{ }^{\circ} \mathrm{C}$ during 20 minutes.

\subsection{Organic photodetector characterization}

4.2.1 EQE measurements. Materials and devices. The EQE setup consists of a Supercontinuum White laser (Fianium PM-SC) coupled to a Fianium monochromator (LLTFContrastTM), with the power calibrated by a broad-band silicon photodetector (Thorlabs S120 V 200-1100 nm $50 \mathrm{~mW}$ ). The setup is enclosed on a black box, to prevent any light from affecting the measurement. The EQE wavelength scan was done from $400 \mathrm{~nm}$ to $1100 \mathrm{~nm}$ every $2.8 \mathrm{~nm}$ followed by a no-light measurement of the dark current of each device. With the same setup and procedure, we measured EQE under reversed $\mathrm{V}$ bias up to $28 \mathrm{~V}$. The electric characterization was performed with a Keythley 2450 SourceMeter ${ }^{\circledR}$.

4.2.2 Linear dynamic range measurement. The linear dynamic range of our devices was measured with the aforementioned EQE setup at $907 \mathrm{~nm}$ where one of the major enhancement peaks of our photodetectors occur. The power of the laser was changed manually and measured every time to perform the sweep.

4.2.3 Transient photocurrent measurements. The transient photocurrent measurements were performed with the EQE set up fixed at $907 \mathrm{~nm}$ and chopped at $500 \mathrm{~Hz}$ with a mechanical chopper.

4.2.4 LBIC measurements. The white laser and monochromator setup was connected to an optic fiber and a beam splitter. This setup enables simultaneous measurement of the incident power and device power output. The beam impinged onto the sample through a microscope $(20 \times)$. The sample was mounted onto a XYZ stage (TDC001 - T-Cube DC Servo Motor Controller and MTS50-Z8 Mounted in 3-Axis XYZ configuration). In the procedure, the sample was moved along the $X Y$ plane to extract the photocurrent maps. The whole system was controlled with LabView 2018 developed software and the electrical response was measured with a Keythley 2450 SourceMeter ${ }^{\circledR}$.

4.2.5 FDTD design. The numerical calculations were performed using Lumerical FDTD solutions (www.lumerical.com). The simulated architecture reproduced the schematic shown in Fig. 4 with the geometrical parameters extracted from SEM micrographs of the samples. A linearly polarized plane wave source impinging a unit cell was modelled providing a good agreement with experimental results. Refractive indexes of the PBTTT:PC ${ }_{71} \mathrm{BM}$ were extracted from ellipsometry (GES5E from Sopra).

4.2.6 SEM characterization. SEM images were obtained with a Scanning Electron Microscope (SEM) (SEM Quanta 650 FEG) at $5 \mathrm{kV}$ to generate secondary electrons in a high vacuum regime.

\section{Conflicts of interest}

The authors declare no conflicts of interest. 


\section{Acknowledgements}

We greatly acknowledge financial support from the Ministerio de Ciencia, Innovación y Universidades MICINN with projects PGC2018-095411-B-I00, MAT2016-79053-P and MAT2015-70850$\mathrm{P}$ and the "Severo Ochoa" excellence program SEV-2015-0496; Generalitat de Catalunya program AGAUR 2017-SGR-00488; and the European Research Council (ERC) under the European Union's Horizon 2020 research and innovation programme (grants no. CoG648901 and StG637116). P. M. acknowledges financial support from an FPI contract (2017) of the MICINN (Spain) cofounded by the ESF. M. G. R. acknowledges financial support from an FPU grant (no. 16/02631) (2017) of the MICINN (Spain). M. G. R. and P. M. B. acknowledge the departments of Physics, Chemistry and Geology of the Autonomous University of Barcelona (UAB) as coordinators of the PhD programme in Materials Science. We acknowledge support of the publication fee by the CSIC Open Access Publication Support Initiative through its Unit of Information Resources for Research (URICI).

\section{References}

1 X. Gong, M. Tong, Y. Xia, W. Cai, J. S. Moon, Y. Cao, G. Yu, C. L. Shieh, B. Nilsson and A. J. Heeger, Science, 2009, 325, 1665-1667.

2 H. Wang and D. H. Kim, Chem. Soc. Rev., 2017, 46, 5204-5236.

3 R. J. Bruening, Appl. Opt., 1987, 26, 1051-1057.

4 L. R. Canfield, R. E. Vest, T. N. Woods and R. S. Korde, Proc. SPIE, 1994, 2282, 31-38.

5 R. R. Søndergaard, M. Hösel and F. C. Krebs, J. Polym. Sci., Part B: Polym. Phys., 2013, 51, 16-34.

6 C. Brabec (Linz) and E. Hauch (Erlangen), US Pat., 7476278 B2, 2009.

7 S. Tong, J. Yuan, C. Zhang, C. Wang, B. Liu, J. Shen, H. Xia, Y. Zou, H. Xie, J. Sun, S. Xiao, J. He, Y. Gao and J. Yang, npj Flexible Electron., 2018, 2, 1-8.

8 T. Yokota, P. Zalar, M. Kaltenbrunner, H. Jinno, N. Matsuhisa, H. Kitanosako, Y. Tachibana, W. Yukita, M. Koizumi and T. Someya, Sci. Adv., 2016, 2, 1-9.

9 Y. Li, Acc. Chem. Res., 2012, 45, 723-733.

10 M. R. Antognazza, U. Scherf, P. Monti and G. Lanzani, Appl. Phys. Lett., 2007, 90, 21-24.

11 N. Strobel, N. Droseros, W. Köntges, M. Seiberlich, M. Pietsch, S. Schlisske, F. Lindheimer, R. R. Schröder, U. Lemmer, M. Pfannmöller, N. Banerji and G. HernandezSosa, Adv. Mater., 2020, 32, 1908258.

12 L. Dou, Y. Liu, Z. Hong, G. Li and Y. Yang, Chem. Rev., 2015, 115, 12633-12665.

13 X. Liu, Y. Lin, Y. Liao, J. Wu and Y. Zheng, J. Mater. Chem. C, 2018, 6, 3499-3513.

14 C. Battaglia, C. M. Hsu, K. Söderström, J. Escarré, F. J. Haug, M. Charrière, M. Boccard, M. Despeisse, D. T. L. Alexander, M. Cantoni, Y. Cui and C. Ballif, ACS Nano, 2012, 6, 2790-2797.

15 G. Konstantatos and E. H. Sargent, Nat. Nanotechnol., 2010, 5, 391-400.

16 A. Armin, R. D. Jansen-Van Vuuren, N. Kopidakis, P. L. Burn and P. Meredith, Nat. Commun., 2015, 6, 6343.
17 E. T. Hoke, K. Vandewal, J. A. Bartelt, W. R. Mateker, J. D. Douglas, R. Noriega, K. R. Graham, J. M. J. Fréchet, A. Salleo and M. D. Mcgehee, Adv. Energy Mater., 2013, 3, 220-230.

18 K. Vandewal, K. Tvingstedt, A. Gadisa, O. Inganäs and J. V. Manca, Nat. Mater., 2009, 8, 904-909.

19 C. Deibel, T. Strobel and V. Dyakonov, Adv. Mater., 2010, 22, 4097-4111.

20 P. Panda, D. Veldman, J. Sweelssen, J. J. A. M. Bastiaansen, B. M. W. Langeveld-Voss and S. C. J. Meskers, J. Phys. Chem. $B, 2007,111,5076-5081$.

21 C. M. Yang, P. Y. Tsai, S. F. Horng, K. C. Lee, S. R. Tzeng, H. F. Meng, J. T. Shy and C. F. Shu, Appl. Phys. Lett., 2008, 92, 7-10.

22 H. Chalabi, D. Schoen and M. L. Brongersma, Nano Lett., 2014, 14, 1374-1380.

23 S. Li, D. Xue, W. Xu, Y. Feng, J. Wang, G. Zhang, X. Meng, C. Wang, Y. Song and C. Shu, J. Mater. Chem. C, 2014, 2, 1500-1504.

24 A. G. MacEdo, F. Zanetti, A. Mikowski, J. C. Hummelen, C. M. Lepienski, M. G. E. Da Luz and L. S. Roman, J. Appl. Phys., 2008, 104, 033714.

25 Z. Tang, Z. Ma, A. Sánchez-Díaz, S. Ullbrich, Y. Liu, B. Siegmund, A. Mischok, K. Leo, M. Campoy-Quiles, W. Li and K. Vandewal, Adv. Mater., 2017, 29, 1-8.

26 E. Yablonovitch, J. Mod. Opt., 1994, 41, 173-194.

27 S. W. Baek, P. Molet, M. J. Choi, M. Biondi, O. Ouellette, J. Fan, S. Hoogland, F. P. García de Arquer, A. Mihi and E. H. Sargent, Adv. Mater., 2019, 31, 1901745.

28 P. Shen, G. Wang, B. Kang, W. Guo and L. Shen, ACS Appl. Mater. Interfaces, 2018, 10, 6513-6520.

29 L. Xu, C. Aumaitre, Y. Kervella, G. Lapertot, C. RodríguezSeco, E. Palomares, R. Demadrille and P. Reiss, Adv. Funct. Mater., 2018, 28, 1706291.

30 M. Mariano, G. Kozyreff, L. G. Gerling, P. Romero-Gomez, J. Puigdollers, J. Bravo-Abad and J. Martorell, Light Sci. Appl., 2016, 5, e16216-e16216.

31 Y. Xia and G. M. Whitesides, Angew. Chem., Int. Ed., 1998, 37, 550-575.

32 Y. Xia, J. A. Rogers, K. E. Paul and G. M. Whitesides, Chem. Rev., 1999, 99, 1823-1848.

33 I. McCulloch, M. Heeney, C. Bailey, K. Genevicius, I. MacDonald, M. Shkunov, D. Sparrowe, S. Tierney, R. Wagner, W. Zhang, M. L. Chabinyc, R. J. Kline, M. D. McGehee and M. F. Toney, Nat. Mater., 2006, 5, 328-333.

34 I. C. Ghosekar and G. C. Patil, Microelectron. Eng., 2020, 221, 111195.

35 J. Munshi and G. Balasubramanian, Soft Mater., 2020, 1-14. 36 W. Zhang, H. Shen, B. W. Guralnick, B. J. Kirby, N. A. Nguyen, R. Remy, C. F. Majkrzak and M. E. Mackay, Sol. Energy Mater. Sol. Cells, 2016, 155, 387-396.

37 Y. Yao, Y. Liang, V. Shrotriya, S. Xiao, L. Yu and Y. Yang, Adv. Mater., 2007, 19, 3979-3983.

38 H. L. Zhu, W. C. H. Choy, W. E. I. Sha and X. Ren, Adv. Opt. Mater., 2014, 2, 1082-1089.

39 M. Jahnel, M. Thomschke, S. Ullbrich, K. Fehse, J. D. An, H. Park, K. Leo, C. Im and V. Kirchhoff, Microelectron. Eng., 2016, 152, 20-25. 